\title{
Empirical Analysis on the Fluctuation of Increasing Rate of China's Energy Consumption
}

\author{
Beibei Hu, Xiangdong Song, Zhe Ming, Shuli Wang \& Jierong Yang \\ College of Science, Yanshan University, Qinhuangdao 066004, China \\ E-mail: xizixinxiang@163.com
}

\begin{abstract}
The article made an empirical econometric analysis on the fluctuation of China's total Energy Consumption and then estimated GARCH model, TARCH model and EGARCH model by the way of maximum-likehood estimation. Empirical studies showed GARCH model made the best estimation in statistics and the fluctuation rate was altering. And the fluctuation's impact on China's energy consumption was very high. The TGARCH and EGARCH models also showed that it had asymmetrical relation with the fluctuation of China's total energy consumption. The authors summarized the features of volatility from empirical results and put forward some suggestions for further development and improvement.
\end{abstract}

Keywords: Energy consumption, ARCH model, GARCH model, Volatility, Asymmetric

Chinese Library Classification: F224.9 Document code: A

\section{Introduction}

In 1960s, Fama observed the phenomenon of Volatility Clustering which existed in the changes of speculative price and yield. It displayed as: stochastic disturbance term often fluctuates substantially followed by relatively substantial fluctuation and narrower fluctuation was followed by narrower fluctuation. Therefore, many models appeared surrounding GARCH, among which ARCH model was put forward firstly by Robert•Engle which was variance model most successfully simulated changes over time. The core idea of ARCH was described as follows: the variance of errors was described by an autoregressive model and it was stochastic volatility no longer but characters with 'memory'. Later, Bollerler, Engle, Lilien, Rbbins etc. modified the ARCH model successively and put forward a series of generalized model such as GARCH, TARCH, EGARCH and so on. These models including $\mathrm{ARCH}$ model made up a set of relatively complete conditional heteroscedastical autoregressive theory whose most important application was to study volatility clustering through heteroscedasticity.

In the paper, the authors made an empirical analysis on the Volatility of growth rate of total energy consumption in China using the family of ARCH models, which mainly discussed whether the growth rate of total energy consumption showed asymmetry. In fact we may imagine that the asymmetry exists because there were many mechanisms playing roles in the fluctuation of China's total energy, in which the most obvious external shock mechanisms included policies shock, price shock, climate impact and so on. They made a difference on the production and consumption of China's energy simultaneously. Due to all the uncertain factors above there would be substantial fluctuation about China's total energy consumption in some years. The method we adopted was to prove the sustainability of volatility rate using GARCH etc. models under the hypothesis that it had asymmetrical relation between growth rate and fluctuation rate. Looking from research purpose, on the basis that GARCH model could make better estimation, TARCH model or EGARCH model would provide better estimation for the growth of China's energy consumption than GARCH model, which would support the asymmetry. If GARCH model products better empirical results but TARCH or EGARCH model don't, this would support the symmetry.

\section{Basic idea}

\subsection{ARCH model}

The autoregressive conditional heteroskedasticity $(\mathrm{ARCH})$ model was widely used to study the kind of the problem of heteroskedasticity. The model was represented as shown below.

Established the existence of a set of regression equation

$y_{t}=r_{0}+r_{1} x_{1 t}+u_{t}$ 


$$
u_{t} \sim N\left(0, \sigma_{t}^{2}\right)
$$

$$
\sigma_{t}^{2}=a_{0}+a_{1} u_{t-1}^{2}
$$

The equation (1) is a basic regression equation; the equation (20 shows the error $u_{t}$ obeys normal distributions with expectation 0 and variance $\sigma_{t}^{2}$; the equation (3) shows the variance $\sigma_{t}^{2}$ of $u_{t}$ consists of two parts: a constant and squared residuals in the former time. The regression model with all the characters above will be called ARCH model.

\subsection{GARCH model}

In 1986, Bollasler put forward the generalized form of ARCH model which was Generlized Autoregerssive Conditional Heteroskedasticity $(\mathrm{GARCH})$. This was proved it was a very valuable step for practical work. The conditional variances of GARCH model are shown below:

$$
\sigma_{t}^{2}=a_{0}+\sum_{i=1}^{q} \alpha_{i} u_{t-i}^{2}+\sum_{j=1}^{p} \beta_{j} \sigma_{t-j}^{2}
$$

To make sure the conditional variance $\sigma_{t}^{2}>0$, we require $\alpha_{i}>0, \beta_{j}>0, i, j=1,2, \ldots . n$ and use $\operatorname{GARCH}(p, q)$ to express GARCH process with order $\mathrm{p}$ and $\mathrm{q}$. To make sure $\operatorname{GARCH}(p, q)$ is steady there is restrict $\sum_{i=1}^{q} \alpha_{i}+\sum_{j=1}^{p} \beta_{j}<1$. The simplest GARCH model is GARCH $(1,1)$ model: $\sigma_{t}^{2}=a_{0}+\alpha_{1} u_{t-1}^{2}+\beta_{1} \sigma_{t-1}^{2}$

\subsection{TARCH model}

TARCH model was put forward by Zakoian and Runkle to deal with asymmetric GARCH model. The conditional variances of TARCH model has following form:

$$
\sigma_{t}^{2}=a_{0}+\alpha u_{t-1}^{2}+\gamma d_{t-1} u_{t-1}^{2}+\beta \sigma_{t-1}^{2}
$$

In the equation, $d_{t-1}$ is nominal variable $d_{t-1}=\left\{\begin{array}{l}1, u_{t-1}<0 \\ 0, u_{t-1} \geq 0\end{array}\right.$

If $\gamma>0$, we will say there is leverage effect and the main effectiveness of asymmetric effect is to make the fluctuation increase. If $\lambda<0$, the function of asymmetric effect is to make the fluctuation decrease.

\subsection{EGARCH model}

EGARCH model is to describe the symmetry of the reaction of conditional variance to positive and negative interference in the market. The conditional variances of EGARCH model has following form:

$$
\log \left(\sigma_{t}^{2}\right)=a_{0}+\alpha\left|\frac{u_{t-1}}{\sigma_{t-1}}\right|+\gamma \frac{u_{t-1}}{\sigma_{t-1}}+\beta \log \left(\sigma_{t-1}^{2}\right)
$$

If $\gamma \neq 0$, there will be asymmetry and the leverage effect is obvious. Because of log transformation, it is impossible to have negative variance. The impact of proximate residual is also not binomial but exponential. In addition variance is represented in exponential form so there is no any restrict for the parameter in the model which is the great advantage of EGARCH model.

\section{Empirical analysis}

\subsection{The selection of data and basic statistical analysis}

The sampled data adopted in the article are China's total energy consumption (NY) in recent years. The time span of data is from 1953 to 2007 and the data source from China statistical yearbook 2008 and the recent years' China statistical yearbook. Since the founding of PR China our total energy consumption is increasing year by year. To eliminate variable-considerable fluctuation we calculate the increasing rate of China's total energy consumption according to the formula $\left(y_{t}=\frac{x_{t}-x_{t-1}}{x_{t-1}}\right)$ and obtain the series $y_{t}$ of increasing rate from 1953 
to 2007. Here econometric software Eviews (5.0) was mainly used to deal with data. The statistics of the series of annual rate of growth of China's energy and the increasing rate of China's total energy consumption from 1953 to 2007 is described in figure 1 and table 1 respectively.

The Figure 1 shows there are obvious characters of 'volatility clustering' in the series $y_{t}$ of China's energy, which means that the former volatility has remarkable impact on the system and the sharp fluctuation concentrates on some periods of time, so the narrow fluctuation does. From the figure we directly see the fluctuation rate of the series $y_{t}$ in the former period of time is bigger than the subsequent period of time and the time set apart is consistent with the time of our country's reforms. As is shown in the table 1, the series $y_{t}$ is not equal to 0 obviously and the range value between the Maximum and Minimum is comparatively large. The fluctuation is also very big seen from the Standard deviation. The Skewness value is positive and shows the character of fluctuation skewed to the right.

\section{2 the establishment of the set of ARCH models.}

Before establishing ARCH model, we must verify the stability of the time series $y_{t}$ firstly. This article analyze whether there is unit root in actual growth rate using ADF and PP tests because only the actual growth rate is stable we could obtain reliable parameter estimation and statistical inference. We take the unit root in three situations: the auxiliary regression including intercept, the auxiliary regression including time trend and intercept and the auxiliary regression not including any term. The result is shown in Table 2.

From Table 2 we can know the test statistics of series $y_{t}$ are less than the critical values at 0.01 significant level in all kinds of situations so there is no root unit at $95 \%$ confidence level and we should reject the null hypothesis, which means the series $y_{t}$ is stable.

(1) GARCH $(1,1)$ model estimation is shown in table 3 and the conditional mean and the conditional variance can be set as follows:

$$
\begin{aligned}
& y_{t}=r_{0}+\sum_{i=1}^{k} r_{i} y_{t-i}+u_{t} \quad k=1 \\
& \sigma^{2}=a_{0}+\alpha u_{t-1}^{2}+\beta \sigma_{t-1}^{2}
\end{aligned}
$$

From $\operatorname{GARCH}(1,1)$ we can see the ARCH and GARCH are significant at $5 \%$ level. The coefficient of ARCH is -0.079919 and the corresponding standard error is 0.003475 ; the coefficient of GARCH is 1.017231 and the corresponding standard error is 0.005652 but the sustainability index which reflects the fluctuation of the system is $(\alpha+\beta)=0.937312$. So we can see that the impact of fluctuation on China's total energy consumption is very great.

(2) TARCH (1,) model estimation is shown in table 4. The conditional mean and the conditional variance are set as follows:

$$
\begin{aligned}
& y_{t}=r_{0}+\sum_{i=1}^{k} r_{i} y_{t-i}+u_{t} \quad k=1 \\
& \sigma_{t}^{2}=a_{0}+\alpha u_{t-1}^{2}+\gamma d_{t-1} u_{t-1}^{2}+\beta \sigma_{t-1}^{2}
\end{aligned}
$$

From table 4 we can know the coefficient $\gamma=-0.040970$; the standard error is 0.051219 and it is not significant at $1 \%$ level, which shows fully not only there are no significant differences between the systematic growth of macroscopic energy and the fluctuation intensities in the period of deflation but the systematic fluctuation demonstrates asymmetry and the function of the asymmetry reduces the fluctuation.

(3) EGARCH $(1,1)$ model estimation can be shown in table 5 . The conditional mean and the conditional can be set as follows:

$$
\begin{gathered}
y_{t}=r_{0}+\sum_{i=1}^{k} r_{i} y_{t-i}+u_{t} \quad k=1 \\
\log \left(\sigma_{t}^{2}\right)=a_{0}+\alpha\left|\frac{u_{t-1}}{\sigma_{t-1}}\right|+\gamma \frac{u_{t-1}}{\sigma_{t-1}}+\beta \log \left(\sigma_{t-1}^{2}\right)
\end{gathered}
$$

From table 5 we can see the coefficient of asymmetry is $\gamma=-1.607716$ and it is less than 0 obviously. This demonstrates remarkably there is leverage effect in the systematic fluctuation of energy consumption which 
means the bad news produces larger fluctuation than equal good news.

\section{Conclusion and suggestions}

Empirical study results show GARCH model is remarkable but the TARCH model and EGARCH model are not. In the aspect of data fitting $\operatorname{GARCH}(1,1)$ takes effective estimation in data. By introducing TARCH model and EGARCH model and empirical analysis, the hypothesis that there is asymmetry between growth rate and fluctuation rate is further verified. Therefore the theoretical results are consistent with the practical experience. In addition, from the estimation results of table 3, 4, and 5, the value of sustainability of fluctuation is less than 1 , the results of growth series through unit root test are stable, which verifies each other. Form $\operatorname{GARCH}(1,1)$ model estimation we can know: Firstly, $\beta>1$ means the system of our country's total energy consumption can magnify the former fluctuation itself, but it is impossible for energy consumption to reach stable state relying on the forces of the market. Only through non-market inference from exterior powers the stability of the system can be realized. Secondly, $\alpha<0$ shows the external shocks in the former period of time will increase the systematic fluctuation in this period of time and the effect of external governmental inference has an overall function to stabilize the energy consumption system.

With the expand of economies of scale, the China's total energy consumption shows a rising trend and the pressure of energy supply may be large so the fluctuation of energy growth will continue for a long period of time. How to push the energy intensity decline through adjusting economic structure, how to adjust the composition of energy structure so as to support the economy's sustained growth in future with as little energy as possible and how to expand energy supply channels to satisfy Chinese energy need have been very urgent and strategic issues. Empirical research shows the impact of the fluctuation shocks on China's total energy consumption is very great. Therefore, on the one hand, we must improve the potentials of system innovation and technology importation to urge to the improvement of the efficiency our country's energy, so that the fluctuation of the growth of our country's total energy consumption decreases gradually on the basis of perfecting market economy system progressively, expanding the degree of opening up to the outside world and catching up with the advanced international levels in management methods and technology level. On the other hand, we should give full play to the role of exterior government interference. Under the pressure of global greenhouse gas emission reduction each country is facing adjustment of energy strategy and energy policy. Therefore, when making energy policy about greenhouse gas emission reduction our country must take fully its possible impact on energy consumption into account in order to avoid its indirect impact on our country's economic growth target while maintaining our country's total energy consumption to sustainable and steady growth.

\section{References}

Cao, Weilong. (2006). Empirical analysis on the Chinese stock market volatility using ARCH model. World economy. 2006. (1).

Gao, Tiemei. (2006). Econometric analysis approach and modeling: the application and example of Eviews. Beijing: Tsinghua University Press.

Han, Zhiyong, Wei, Yiming, Jiao, Jianling. etc. (2004). On the Cointegration and Causality between Chinese GDP and Energy Consumption. SYSTEMS ENGINEERING. 2004. (12).

Lan, Chunxiang \& Lu, Zhengnan. (2000). The prediction of energy consumption and the choice of energy development strategy in China. Journal of Jiangsu University of Science and Technology(Social Science edition). 2000, (2).

Ma, Hongwei, Wang, Xiaohua \& He, Zuyin. etc. (2006). Empirical analysis on China's energy consumption and economic growth. Business research. 2006(16).

Ma, Xiaowei. (2007). Research on Interaction between Economy Development and Energy Consumption in Our Country. Energy of China. 2007. (5).

National Bureau of Statistics. (2008). China Statistical Yearbook 2008. Beijing: China Statistical Publishing House.

Pang, Hao. (2007). Econometrics. Beijing: Sciences Press.

Yang, Shuping. (2009). Analysis on endogenous volatility in our country's Macroeconomic system based on ARCH Model. Statistics and Decision. 2009. (15). 
Table 1. the statistical description of the growth rate of China's total energy consumption

\begin{tabular}{|c|c|c|c|c|c|c|c|}
\hline mean & Median & Maximum & Minimum & Standard error & Skewness & Peakness & J-B \\
\hline 0.0837 & 0.0664 & 0.8249 & -0.3246 & 0.1476 & 2.0772 & 14.0578 & 313.95 \\
\hline
\end{tabular}

Table 2. The unit root test of the increasing rate of China's total energy consumption

\begin{tabular}{|c|c|c|c|c|c|}
\hline \multirow{3}{*}{ model } & The terms included in the test & Test value & $1 \%$ test value & $5 \%$ test value & $10 \%$ test value \\
\hline \multirow{3}{*}{ ADF } & Intercept & -3.9216 & -3.5627 & -2.9187 & -2.5972 \\
\cline { 2 - 6 } & Trend\&Intercept & -4.0283 & -4.1445 & -3.4986 & -3.1786 \\
\cline { 2 - 6 } & None & -4.1691 & -2.6110 & -1.9474 & -1.6127 \\
\hline \multirow{2}{*}{$\begin{array}{c}\text { PP unit } \\
\text { root }\end{array}$} & Intercept & -4.2037 & -3.5600 & -2.9177 & -2.5967 \\
\cline { 2 - 6 } test & Trend\&Intercept & -4.2894 & -4.1409 & -3.4969 & -3.1776 \\
\cline { 2 - 6 } & None & -3.8015 & -2.6093 & -1.9471 & -1.6129 \\
\hline
\end{tabular}

Table 3. $\operatorname{GARCH}(1,1)$ model estimation

\begin{tabular}{|c|c|c|c|c|}
\hline Statistical Indicator & Coefficient & Std. Error & Z-Statistic & Prob. \\
\hline C & 0.435116 & 0.281668 & 1.544784 & 0.1224 \\
\hline ARCH(1) & -0.079919 & 0.003475 & -22.99800 & 0.0000 \\
\hline GARCH(1) & 1.017231 & 0.005652 & 179.9861 & 0.0000 \\
\hline R-squared & 0.149041 & Mean dependent var & 8.188679 \\
\hline Adjusted R-squared & 0.078128 & S.D. dependent var & 14.80911 \\
\hline S.E.of refression & 14.21885 & Akaike info criterion & 6.578880 \\
\hline Sum squared resid & 9704.434 & Schwarz criterion & 6.764756 \\
\hline Log likelihood & -169.3403 & Durbin-Watson stat & 2.170900 \\
\hline
\end{tabular}


Table 4. TARCH $(1,1)$ model estimation

\begin{tabular}{|c|c|c|c|c|}
\hline Statistical Indicator & Coefficient & Std.Error & Z-Statistic & Prob. \\
\hline C & 0.571562 & 0.382203 & 1.495441 & 0.1348 \\
\hline ARCH(1) & -0.064559 & 0.027782 & -2.323817 & 0.0201 \\
\hline$\gamma$ & -0.040970 & 0.051291 & -0.798781 & 0.4244 \\
\hline GARCH(1) & 1.010124 & 0.048038 & 21.02778 & 0.0000 \\
\hline R-squared & 0.142182 & Mean dependent var & 8.188679 \\
\hline Adjusted & 0.050925 & S.D. dependent var & 14.80911 \\
\hline S.E.of refression & 14.42711 & Akaike info criterion & 6.630599 \\
\hline Sum squared resid & 9782.649 & Schwarz criterion & 6.853651 \\
\hline Log likelihood & -169.7109 & Durbin-Watson stat & 2.222511 \\
\hline
\end{tabular}

Table 5. EGARCH $(1,1)$ model estimation

\begin{tabular}{|c|c|c|c|c|}
\hline Statistical Indicator & Coefficient & Std.Error & Z-Statistic & Prob. \\
\hline $\mathrm{C}$ & 1.020503 & 0.392376 & 2.600832 & 0.0093 \\
\hline$|R E S| / S Q R[G A R C H](1)$ & 0.696497 & 0.234216 & 2.973738 & 0.0029 \\
\hline$\gamma$ & -1.607716 & 0.180331 & -8.915348 & 0.0000 \\
\hline EGARCH( 1) & 0.649631 & 0.070605 & 9.200885 & 0.0000 \\
\hline R-squared & 0.117375 & Mean dependent var & 8.188679 \\
\hline Adjusted & 0.023479 & S.D. dependent var & 14.80911 \\
\hline S.E.of refression & 14.63423 & Akaike info criterion & 7.132186 \\
\hline Sum squared resid & 100065.56 & Schwarz criterion & 7.355237 \\
\hline Log likelihood & -183.0029 & Durbin-Watson stat & 2.269726 \\
\hline
\end{tabular}




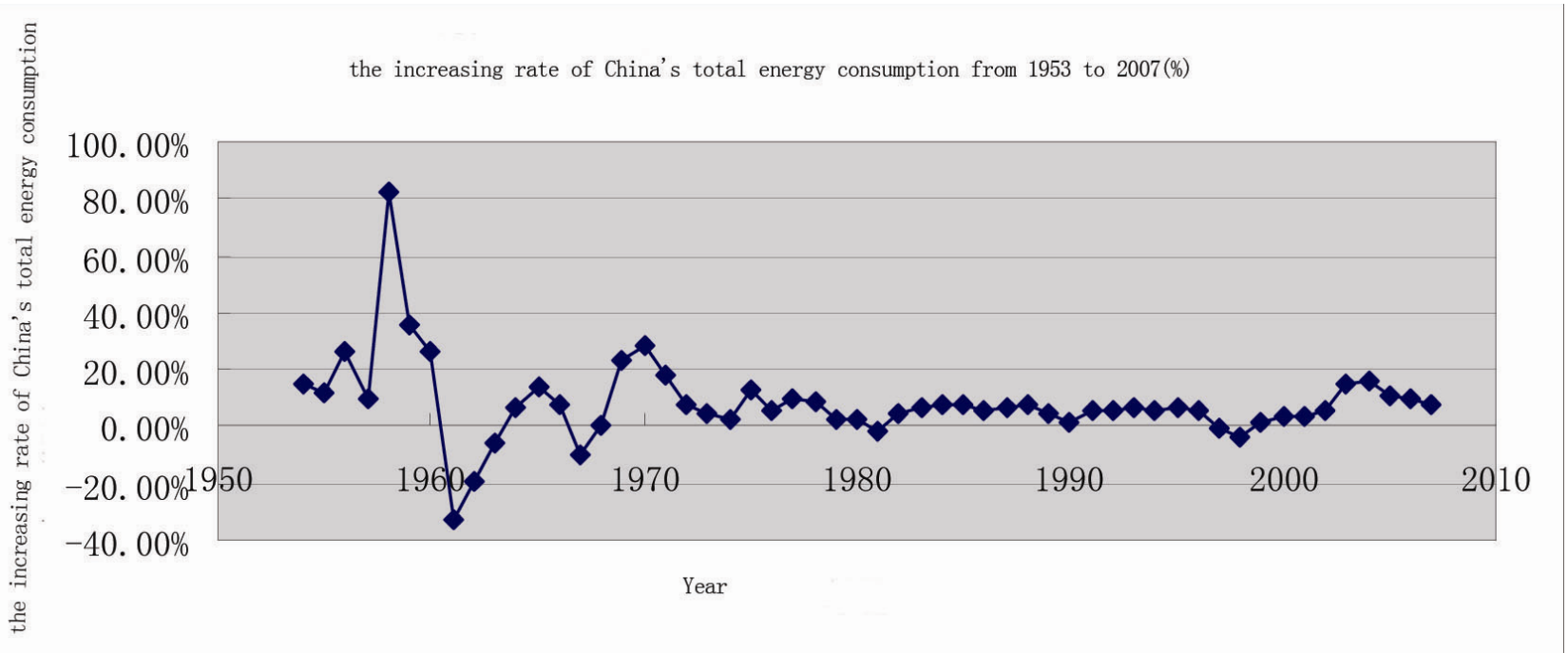

Figure 1. The series of annual rate of growth of China's energy 